\title{
Quantitative Evaluation of Liver Function within MR Imaging
}

\section{Akira Yamada*}

Shinshu University Hospital, Department of Radiology, Matsumoto, Japan

\begin{abstract}
Quantitative evaluation of liver function is important not only for monitoring of that function, but also for preoperative assessment of the liver reserve. Recent advances and future view in quantitative evaluation of liver function within MR imaging was described.
\end{abstract}

\section{Editorial}

Quantitative evaluation of liver function is important not only for monitoring of that function, but also for preoperative assessment of the liver reserve [1].

The Plasma Disappearance Rate of Indocyanince Green (ICGPDR) has been regarded as a valuable tool for the quantitative assessment of liver function, because it is removed from the circulation exclusively by the liver [2]. However a reliable method for the quantitative anatomically based evaluation of segmental liver function has not been established to date, to our knowledge.

The Future Remnant Liver Volume (FRLV) and a quantitative liver function test, such as the ICG clearance test, have been reported to be significant predictors of postoperative liver failure and mortality $[1,3]$. However, with volumetry, precise estimation of the segmental liver reserve could be impossible because the heterogeneity of the liver function could not be taken into account [4].

Gadoxetate disodium is a paramagnetic hepatobiliary contrast agent that can combine the features of extracellular agents with those of a hepatocellular contrast agent. The same transporting mechanisms (i.e. the Organic Anion Transporting Polypeptides, OATPs) are considered to be responsible for uptake of gadoxetate disodium and ICG in hepatocytes; therefore, there is a possibility that gadoxetate disodium-enhanced MR imaging could be the basis of a useful method for quantitative estimation of postoperative liver failure similar to ICG clearance but with anatomic delineation of hepatic function [4-6].

Recent study reported that Relative Contrast Enhancement Index (RCEI) determined from preoperative gadoxetate disodium-enhanced MR imaging can predict Posthepatectomy Liver Failure (PHLF) in patients who underwent major hepatic resection [5]. However, RCEI was determined from the signal intensity of the liver on pre- and post-contrast enhanced MR images without appropriate correction by the vol- ume and the extracellular fluid space of the liver. On the other hand, Yamada et al. [4] reported that the liver function correlating with ICG-PDR can be estimated quantitatively by Hepatocellular Uptake Index (HUI) determined from the signal intensities and the volumes of the liver and spleen on gadoxetate disodium-enhanced MR images with appropriate correction by the volume and the extracellular fluid space of the liver. Because the HUI can correlate with ICG-PDR very well and it can be determined directly from the volume and the signal intensity of a region of interest in the liver, the quantitative estimation of total and segmental liver function may be feasible. Therefore, PHLF could be predicted more precisely by HUI than RCEI, FRLV, or ICGPDR with in gadoxetate disodium-enhanced MR imaging.

In summary, gadoxetate disodium-enhanced MR imaging seems to be a reliable method for the quantitative evaluation of segmental liver function; however, appropriate index and precise limit to avoid PHLF should be determined for the best patient prognosis.

\section{References}

1. Seyama Y, Kokudo N (2009) Assessment of liver function for safe hepatic resection. Hepatol Res 39: 107-116.

2. Sakka SG (2007) Assessing liver function. Curr Opin Crit Care 13: 207-214.

3. Suda K, Ohtsuka M, Ambiru S, Kimura F, Shimizu H, et al. (2009) Risk factors of liver dysfunction after extended hepatic resection in biliary tract malignancies. Am J Surg 197: 752-758.

4. Yamada A, Hara T, Li F, Fujinaga Y, Ueda K, et al. (2011) Quantitative evaluation of liver function with use of gadoxetate disodium-enhanced MR imaging. Radiology 260: 727-733.

5. Cho SH, Kang UR, Kim JD, Han YS, Choi DL (2011) The value of gadoxetate disodium-enhanced MR imaging for predicting posthepatectomy liver failure after major hepatic resection: a preliminary study. Eur J Radiol 80: e195-200.

6. Utsunomiya T, Shimada M, HanaokaJ, Kanamoto M, Ikemoto T, et al. (2012) Possible utility of MRI using Gd-EOB-DTPA for estimating liver functional reserve. J Gastroenterol 47: 470-476.
*Corresponding author: Akira Yamada, Shinshu University Hospital, Department of Radiology, Matsumoto, Japan, Tel: +81-263-37-2650; Fax: +81-263-37-3087; E-mail: a_yamada@shinshu-u.ac.jp

Received April 25, 2012; Accepted April 26, 2012; Published April 28, 2012

Citation: Yamada A (2012) Quantitative Evaluation of Liver Function within MR

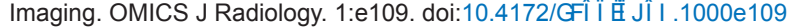

Copyright: (c) 2012 Yamada A. This is an open-access article distributed under the terms of the Creative Commons Attribution License, which permits unrestricted use, distribution, and reproduction in any medium, provided the original author and source are credited. 